\title{
Demo Abstract: PrOLoc: Resilient Localization with Private Observers Using Partial Homomorphic Encryption
}

\author{
Amr Alanwar, Yasser Shoukry, Supriyo Chakraborty \\ Bharathan Balaji, Paul Martin, Paulo Tabuada \\ Mani Srivastava \\ University of California, Berkeley \\ University of California, Los Angeles \\ IBM T. J. Watson Research Lab \\ (alanwar,bbalaji,pdmartin,tabuada,mbs)@ucla.edu \\ yshoukry@eecs.berkeley.edu \\ supriyo@us.ibm.com
}

\begin{abstract}
This demo abstract presents PrOLoc, a localization system that combines partially homomorphic encryption with a new way of structuring the localization problem to enable efficient and accurate computation of a target's location while preserving the privacy of the observers.
\end{abstract}

\section{CCS CONCEPTS}

-Computer systems organization $\rightarrow$ Embedded systems; $R e$ dundancy; Robotics; •Networks $\rightarrow$ Network reliability;

\section{KEYWORDS}

Secure localization, privacy, homomorphic encryption, Paillier cryptosystem

ACM Reference format:

Amr Alanwar, Yasser Shoukry, Supriyo Chakraborty, Bharathan Balaji, Paul Martin, Paulo Tabuada, and Mani Srivastava. 2017. Demo Abstract: PrOLoc: Resilient Localization with Private Observers Using Partial Homomorphic Encryption. In Proceedings of The 16th ACM/IEEE International Conference on Information Processing in Sensor Networks, Pittsburgh, PA USA, April 2017 (IPSN 2017), 2 pages.

DOI: http://dx.doi.org/10.1145/3055031.3055033

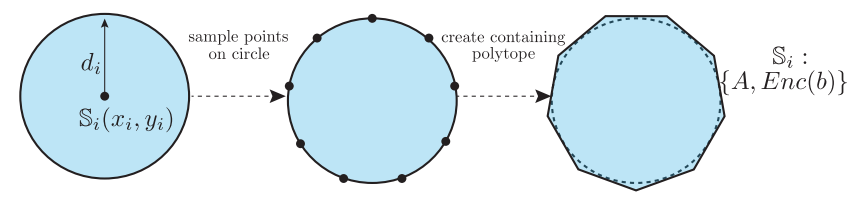

Figure 1: Discretizing the circle as a polyhedron

Permission to make digital or hard copies of all or part of this work for personal or classroom use is granted without fee provided that copies are not made or distributed for profit or commercial advantage and that copies bear this notice and the full citation on the first page. Copyrights for components of this work owned by others than ACM must be honored. Abstracting with credit is permitted. To copy otherwise, or republish, to post on servers or to redistribute to lists, requires prior specific permission and/or a fee. Request permissions from permissions@acm.org.

IPSN 2017, Pittsburgh, PA USA

(c) 2017 ACM. 978-1-4503-4890-4/17/04 ..\$15.00

DOI: http://dx.doi.org/10.1145/3055031.3055033

\section{INTRODUCTION}

Advances in localization techniques have enabled a multitude of services including navigation, targeted advertisements, and locationaware applications. With the growing prevalence of powerful mobile computing devices, techniques for localization both in outdoor and indoor environments are becoming both wide-spread and varied. Many of these localization techniques depend on analyzing various observations of a distinct phenomenon or event-an acoustic chirp, an optical signature, an RF signal, etc-in order to infer the location of some object or event. In such scenarios, measured signals are often captured by a number of sensors or observers and then typically communicated to a centralized computer or an aggregator for post-processing and eventually location estimation.

The knowledge of observers' locations has a potential risk. Such risk appears whenever (1) the observers do not belong to the same trust zone and/or (2) all observers belong to the same trust zone but not the cloud used to perform the localization computations. Usage examples of PrOLoc can be summarized as following:

- Consider a smart car which has lost its GPS signal [3], PrOLoc can allow that car to send requests and get assistance in finding its location from nearby vehicles. In such scenario, the assisting cars belong to different trust zones, and they may not be willing to share their location information for fear of privacy violation. PrOLoc provides privacy guarantees to these vehicles during the localization process.

- In the context of smart cities in which citizens are asked to report safety and maintenance issues to government authorities. In such a scenario, citizens may not trust each other or the government (cloud).

- In a military scenario, soldiers in the field may report the location of events nearby without revealing their location. Although all soldiers belong to the same trust zone (they trust each other), a compromised cloud/aggregator that leaks their information can lead to severe consequences.

Therefore, performing all localization calculations while preserving the privacy of the observers is highly desirable. PrOLoc [2] reformulates the traditional localization while preserving the privacy of anchor nodes. The intuition behind the PrOLoc algorithms

Amr Alanwar and Yasser Shoukry are equally contributing authors. 

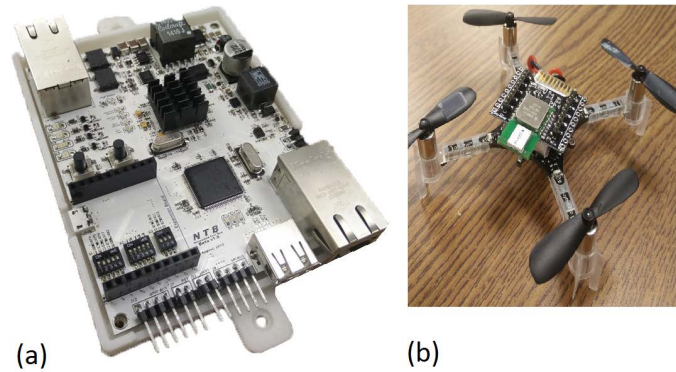

Figure 3: (a) Custom ranging anchor circuit board, and (c) mobile ranging target.
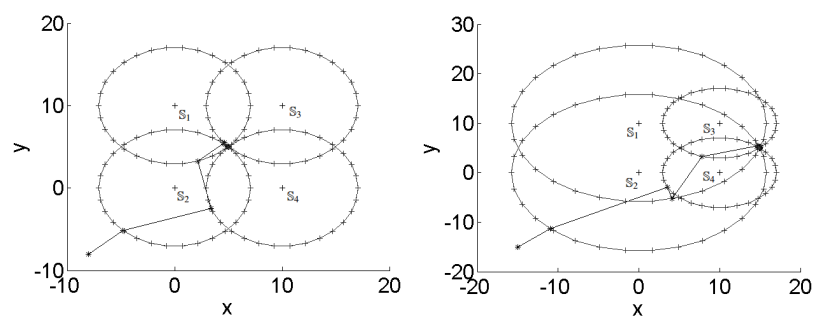

Figure 2: Several iterations of the alternating projection algorithm for different geometric configurations-a target inside (left) and outside (right) the observers' convex hull.

is as follows. Traditional geometry-based localization methods like the least squares approach treats range measurements as an estimate that the target $\mathbb{T}$ lies on some circle whose radius is equal to the estimated distance and whose center is the coordinate of the sensor. In essence, communicating this constraint requires communicating both the radius $\left(d_{i}\right)$ and center $\left(x_{\mathbb{S}_{i}}, y_{\mathbb{S}_{i}}\right)$, exposing sensitive information about the sensors themselves. Rather than parameterizing the sensing range as a circle, we seek another parameterization that can still represent the estimated range without sacrificing sensor privacy. Towards this end, we propose using polyhedra to represent the sensed range. We can obtain such polyhedra by randomly sampling the circumference of the circle representing the measured range, as shown in Figure 1.

Rethinking the polyhedra-based localization algorithm, we can formulate the localization problem to that of finding a point that lies on the boundary of the intersection of all polyhedra generated by observers. Such an algorithm can be applied as follows. Starting from any arbitrary initial estimate, we start by projecting this estimate onto the boundary of the polyhedron $\mathcal{P}_{1}\left(A_{1}, b_{\mathbb{S}_{1}}\right)$ generated by the first observer followed by a projection onto the boundary of the polyhedron $\mathcal{P}_{2}\left(A_{2}, b_{\mathbb{S}_{2}}\right)$ and so on until we project onto all $m$ polyhedra. By repeating the previous sequence of projections until we reach the maximum number of iterations, the alternating projection algorithm [4] guarantees that the estimate converges to the target location. An example of the sequence of points generated by this algorithm is shown in Figure 2 for the two cases when (i) the target is inside the convex hull of the observers and (ii) the target is outside the convex hull of the observers.

\section{DEMONSTRATION}

We use the Paillier cryptosystem and the Java BigInteger library with full floating point support. We implement both the Aggregator and Querier on Macbook Pro laptops with Intel(R) Core i7-4870HQ CPUs @2.5GHz. The target node is constrained to motion on a fixed $z$ plane, so that problem reduces to localization in $2 D$.

Anchor Ranging Hardware: In our demo experiments, the anchor nodes used will consist of custom-built circuit boards equipped with ARM Cortex M4 processors at $196 \mathrm{MHz}$ communicating to Decawave DW1000 ultra-wideband radios, as shown in Figure 3. Each anchor node listens for incoming range messages from a target node and, upon reception, begins a symmetric double-sided ranging sequence. After completing a range sequence, each anchor communicates the range estimate to an Android Nexus 5 smartphone to perform the secure communication to the Aggregator localization server. In effect, the Nexus 5 and custom ranging hardware serve together as a single cohesive observer node.

Mobile Target Ranging Hardware The mobile targets consist of Battery-powered mobile nodes also with ARM Cortex M4 processors based on the CrazyFlie 2.0 helicopter [1] and equipped with the DW1000 radio.

\section{ACKNOWLEDGMENTS}

This research is funded in part by the National Science Foundation under awards \# CNS-1329755 and ACI-1640813, and by the NIH Center of Excellence for Mobile Sensor Data to Knowledge under award \# 1U54EB020404-01. The U.S. Government is authorized to reproduce and distribute reprints for Governmental purposes notwithstanding any copyright notation thereon. The views and conclusions contained herein are those of the authors and should not be interpreted as necessarily representing the official policies or endorsements, either expressed or implied, of NSF, NIH, or the U.S. Government.

\section{REFERENCES}

[1] Bitcraze CrazyFlie 2.0. https://www.bitcraze.io/. Accessed: 2017-01-01.

[2] A. Alnwar, Y. Shoukry, S. Chakraborty, P. Martin, P. Tabuada, and M. Srivastava. PrOLoc: Resilient Localization with Private Observers Using Partial Homomorphic Encryption. In Proceedings of the 16th international conference on Information Processing in Sensor Networks. ACM.

[3] S. U. Hussain and F. Koushanfar. 2016. Privacy preserving localization for smart automotive systems. In 2016 53nd ACM/EDAC/IEEE Design Automation Conference (DAC). 1-6. DOI : http://dx.doi.org/10.1145/2897937.2898071

[4] J. Von Neumann. 1950. Functional Operators: The Geometry of Orthogonal Spaces. Princeton University Press. 